\title{
A Novel approach for Segmentation and Classification of brain MR Images using Cluster Deformable Based Fusion Approach.
}

\author{
Sharan Kumar' ${ }^{1}$, D.Jayadevappa ${ }^{2}$, Mamata V Shetty ${ }^{3}$ \\ ${ }^{1} \mathrm{Ph} . \mathrm{D}$ Scholar Electronics \& Instrumentation Engineering Department, JSS Academy of Technical Education Bangalore, \\ kumar.sharan87@gmail.com \\ ${ }^{2}$ Electronics \& Instrumentation Engineering Department, JSS Academy of Technical Education Bangalore, ${ }^{2}$ devappa22@gmail.com \\ ${ }^{3} \mathrm{Ph} . \mathrm{D}$ Scholar Electronics \& Instrumentation Engineering Department JSS Academy of Technical Education Bangalore
}

\begin{tabular}{l}
\hline Article Info \\
\hline Article history: \\
Received Jun $12^{\text {th }}, 2018$ \\
Revised Aug $20^{\text {th }}, 2018$ \\
Accepted Aug $26^{\text {th }}, 2018$ \\
\hline
\end{tabular}

\section{Keyword:}

Tumorous cells, MRI images, brain tumor classification, Deformable model, clustering

\begin{abstract}
Segmentation of tumor form brain MR images is the most important and tedious task in the medical field. In this paper, A Cluster deformable based fusion approach which uses both deformable and KMeans clustering scheme for Segmentation is discussed. The features of tumor and non tumor cases are extracted with the use of the Power Local Binary Pattern (LBP) Operator after completion of the segmentation process. The extracted features are fed to Naive Bayes classifier to perform the process of classification. Here, the validation of the proposed system is done using standard validation methods such as accuracy, specificity, sensitivity and RoC metrics. The developed method is applied for MR images collected from standard SimBRATS database. Experimentation results shows that the proposed method performs better when compared to the traditional clustering and deformable methods and this scheme got accuracy of $84.8 \%$.
\end{abstract}

\section{Corresponding Author:}

\section{Sharan Kumar,}

${ }^{1} \mathrm{Ph} . \mathrm{D}$ Scholar Electronics \& Instrumentation Engineering Department, JSS Academy of Technical Education Bangalore, kumar.sharan87@gmail.com

\section{Introduction}

Brain tumor may appear in different sizes and types with different image intensities. Some of them may also affect the surrounding structures that change the image intensities around the tumor. As of Now in medical field the clinical methods which are used are of not so accuracy and robustness in detecting the tumor form the MR image of the brain. Appearance of the individual Two Dimensional (2D) MRI slices and scheduling the treatment are the tough jobs for the doctors. The existing algorithms gives a challenge in the research to choose this medical field to choose as the research field and to increase the automation in this field that should lead to accurateness and correctness. Nowadays this field is chosen as the active field for research and many researcher are opting this field

Results in the medical field should be more accurate as this filed is so vey sensitive with respect to results, hence the classification algorithm used in the classification ogf tuors from MR images should be more accurate. As the inaccuracy is not acceptable in the fields like surgical planning, treatment planning since a minor error may lead to many problems and may lead to improper treatment. .

In the last few years, many segmentation algorithms are developed and validated which gives more accuracy but still few algorithms fail to give output under some situations. Segmenting the tumor region from MR brain image is not a simple task, it requires many complex processing. Segmentation is done by considering many features like shape of the 
tumor, Size of the tumor .In MR image of the brain has three segments called White Matter (WM), Gray Matter (GM) and Cerebro-Spinal Fluid (CSF) .

In Recent years automation of medical image processing has taken a major role in solving many problems and giving solutions with in less time and with less probability of errors as manual detection are error prone.

In This Module the major challenges are

i) To design and develop a segmentation algorithm which has the features of both the algorithms like deformable and K-Means clustering

ii) Extract the features of tumor and non tumor cases with the use of the Power Local Binary Pattern (LBP) Operator.

iii) Training the Naive Bayes [NB] classifier to perform the process of classification from the features extracted using Power LBP operator.

\section{Literature Review}

In this section, the existing techniques that contribute to the brain tumor segmentation and classification is elaborated.

\section{Image Segmentation Based on Clustering Model.}

Clustering can be considered as one of the most important unsupervised learning Method.

Types of Clustering:

1. Hierarchical Clustering 2. Partitional Clustering 3. Density-Based Clustering

4. Grid-Based Clustering and 5. Model-Based Clustering.

\begin{tabular}{|l|l|l|l|}
\hline \multicolumn{1}{|c|}{ Authors } & \multicolumn{1}{|c|}{ Contribution } & \multicolumn{1}{c|}{ Advantages } & \multicolumn{1}{c|}{ Disadvantages } \\
\hline $\begin{array}{l}\text { Zhengwang Wu et al. } \\
\text { [1] }\end{array}$ & $\begin{array}{l}\text { learning-based deformable } \\
\text { model }\end{array}$ & $\begin{array}{l}\text { reduces the } \\
\text { computational cost, and } \\
\text { improves the prediction } \\
\text { accuracy }\end{array}$ & $\begin{array}{l}\text { This framework do not } \\
\text { simultaneously segment } \\
\text { multiple Region of } \\
\text { Interests (ROIs) (i.e, with } \\
\text { more brain regions) }\end{array}$ \\
\hline $\begin{array}{l}\text { Shoaib Amin Banday, } \\
\text { and Ajaz Hussain Mir } \\
\text { [2] }\end{array}$ & $\begin{array}{l}\text { A technique which is semi- } \\
\text { automatic }\end{array}$ & $\begin{array}{l}\text { It shows better accuracy, } \\
\text { and is reliable and fast }\end{array}$ & $\begin{array}{l}\text { It consumes a lot of effort } \\
\text { and time to properly } \\
\text { outline abnormalities slice } \\
\text { by slice }\end{array}$ \\
\hline Hala Ali et al. [3] & $\begin{array}{l}\text { wavelet multi-resolution } \\
\text { (WM), morphological } \\
\text { pyramid fusion (M), and } \\
\text { fuzzy C-mean clustering } \\
\text { (FCM) segmentation } \\
\text { system, (WMMFCM) }\end{array}$ & $\begin{array}{l}\text { The accuracy of the } \\
\text { segmentation process } \\
\text { can be enhanced }\end{array}$ & $\begin{array}{l}\text { Noise is the challenging } \\
\text { problem }\end{array}$ \\
\hline $\begin{array}{l}\text { Ali Ahmadvand et al. } \\
\text { [4] }\end{array}$ & $\begin{array}{l}\text { Dynamic Classifier } \\
\text { Selection Markov Random } \\
\text { Field (DCSMRF) algorithm }\end{array}$ & $\begin{array}{l}\text { The accuracy and } \\
\text { reliability are enhanced } \\
\text { by using the ensemble } \\
\text { method }\end{array}$ & $\begin{array}{l}\text { huge computational time } \\
\text { on big datasets with a } \\
\text { complex feature space and } \\
\text { more than two classes }\end{array}$ \\
\hline
\end{tabular}


R.Lavanyadevi, M.Machakowsalya, J.Nivethitha, and A.Niranjil [5] Proposed a methodology in which segmentation is done using K-Means Clustering and the neural network is used as the classifier to classify the brain tumour as benign, malignant or normal. The features are extracted using the Gray Level Co-Occurrence Matrix (GLCM). Here the classifier used is probabilistic neural network (PNN).They have mentioned that PNN is fastest technique and provides the good classification accuracy.

\section{Proposed a Fusion Approach Cluster deformable based fusion for tumor segmenting and classifying in MR} Brain Images.

In the figure shown below explains the clear flow of segmentation and classification process, Segmentation of MR brain Image is done using the fusion approach which has the properties of $\mathrm{K}$ - means clustering algorithm and deformable algorithm, the complete process is as shown in the Figure 1.

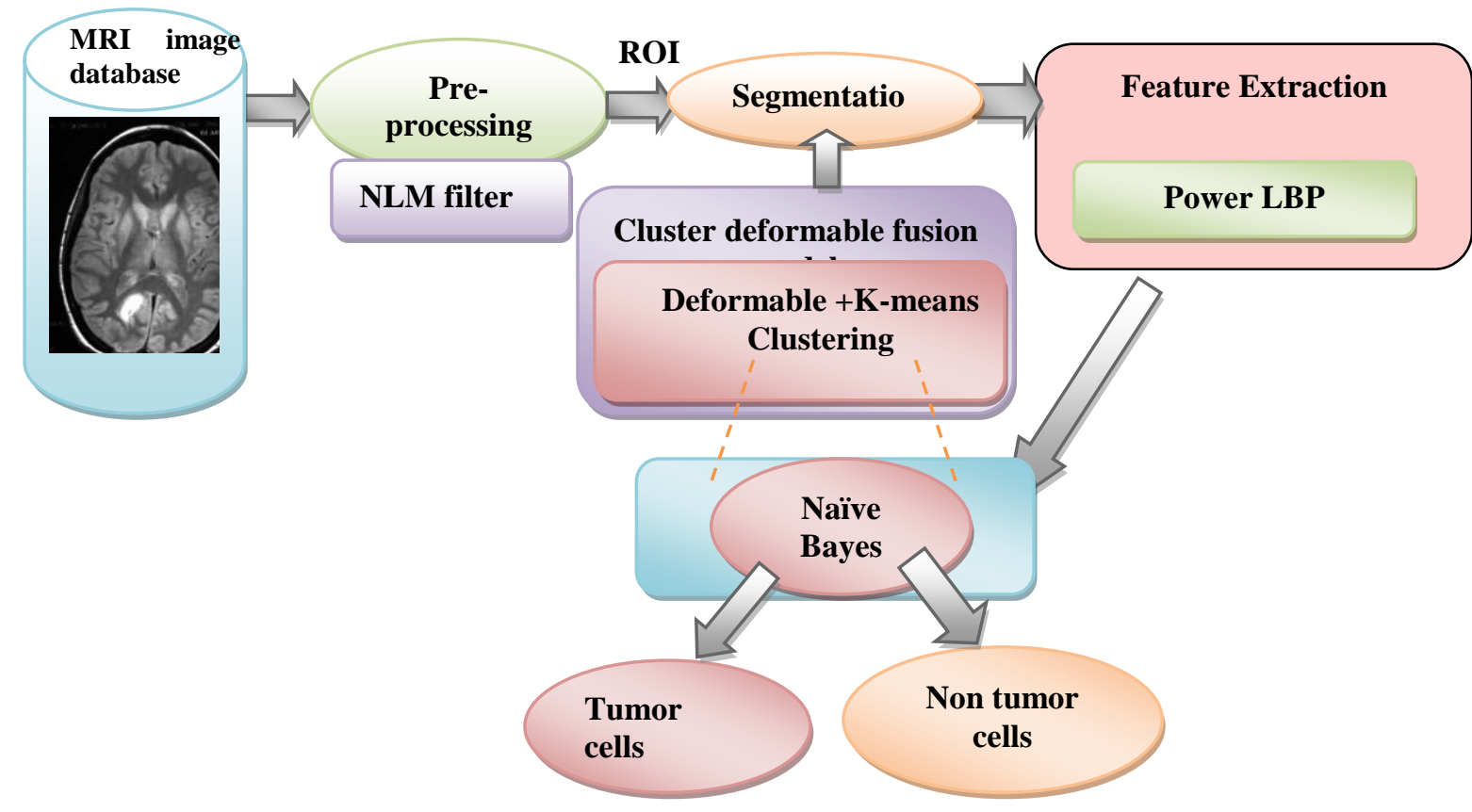

Fig 1 Schematic diagram of Segmentation and classification using Cluster Deformable Based Fusion Approach.

The process includes four main stages as shown in figure 1 namely 1) pre-processing, 2) Segmentation, 3) Feature extraction, and 4) Classification.

- The MR Image of the brain is taken from ther standard SimBRATS database, and pre-processing of the image is done Non Local Means (NLM) filter to extract the required region of interest (ROI).

- Once the ROI is obtained, Segmentation process is carried out, here we use the proposed fusion approach, which is cluster deformable model.

- The proposed scheme uses both the segmentation algorithm which are K-means clustering and deformable model.

- The required features are extracted using Power LBP operator which has the information related to tumor and Non tumor .

- Then the features extracted are given to Naive Bayes Classifier for the classification.

- The Simulation and experimentation is done using the MR images of the brain taken from SimBRATS database.

- Validation of the proposed system is done by using validation method like accuracy, on Specificity, Sensitivity, accuracy and ROC Metrics.

- Experimentation shows that this method has better performance compared to the existing algorithms.

- The Proposed design achieved accuracy of $84.8 \%$. 


\section{Comparative techniques}

Experimentation is done using the propsed segmentation scheme the cluster based deformable fusion approach and after the classification process, the outputs are compared with the existing algorithms. Here two algorithms are used for comparison which are Fuzzy C-means (FCM) + Support Vector Machine (SVM), and Possibilistic Fuzzy C-means algorithm PFCM + k- Nearest Neighbor (k-NN) .The description of these algorithm is as given below.

FCM + SVM: SVM classifier [5], one of the commonly used binary classifiers, performs the classification task, by defining the hyperplane. Here, FCM algorithm is used for the clustering of tumor segments.

PFCM + k-NN: The k-NN algorithm [6] promotes the classification task, by grouping the classification samples into two classes i.e,) $\mathrm{k}=2$. The PFCM technique defined in [4], is used for the tumor segmentation purpose.

\section{Experimental Results}

The experimental results obtained by the developed classification technique are explained in this section, the results obtained at every stage of segmentation of classification using SimBRATS database are as shown here.

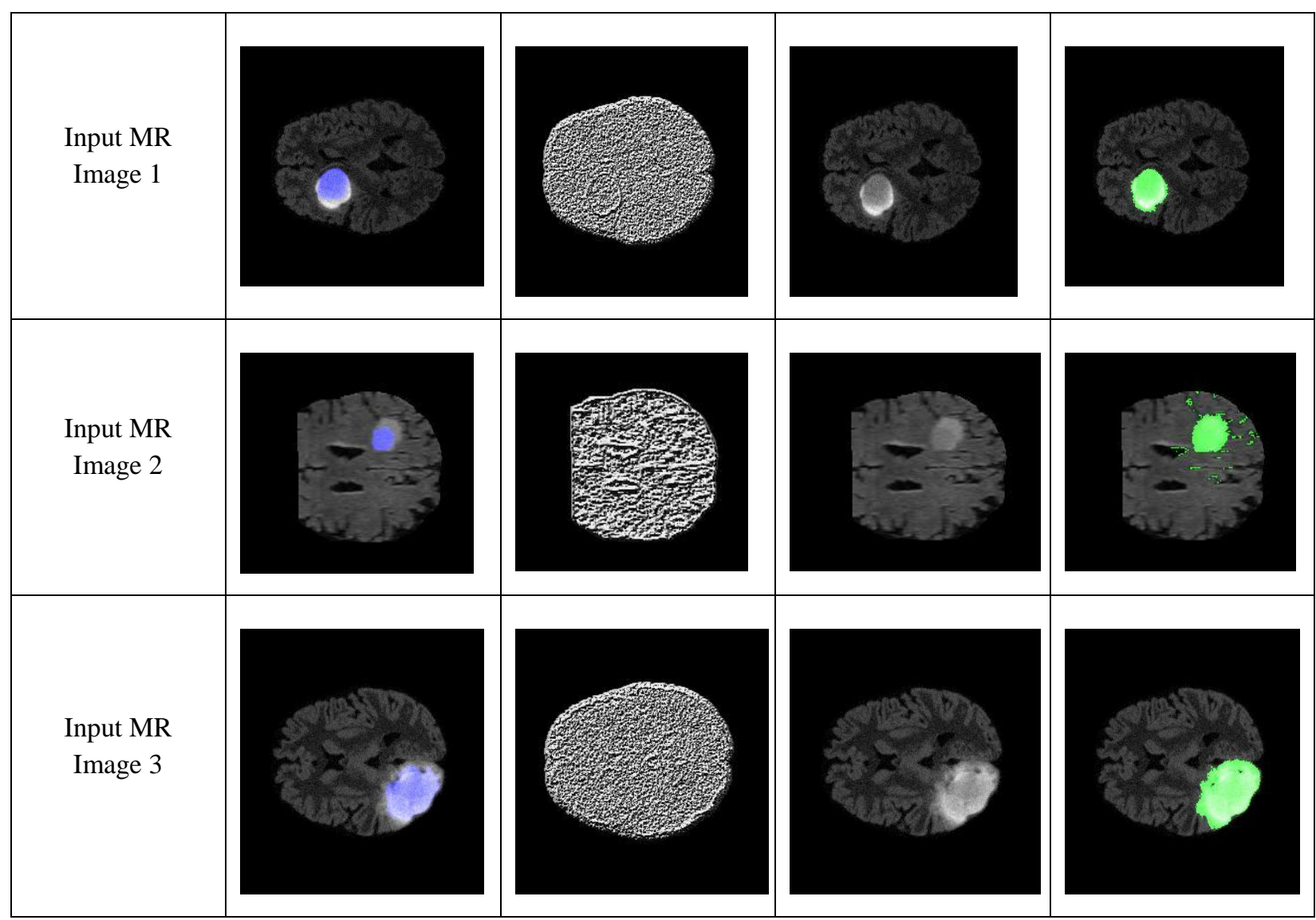

Fig 2. Experimental results of proposed Cluster deformable + NB for SimBRATS database

(a) Ground truth (b) Power LBP feature, (c) Original image, and (d) Classified output.

\subsection{Comparative analysis for SimBRATS database}

\section{i)Analysis by varying the training data}

The comparative analysis of the proposed scheme in SimBRATS database is presented in Table 1, and the analysis is done by varying the training data. 
Table 1: Comparative analysis with SimBRATS database for varying training data based on (a) Sensitivity, (b) specificity, and (c) accuracy using proposed Cluster deformable based fusion approach + NB For $90 \%$ of training data

\begin{tabular}{|c|c|c|c|}
\hline \multirow{2}{*}{$\begin{array}{c}\text { Techniques used for } \\
\text { Comparison }\end{array}$} & \multicolumn{3}{|c|}{ Evaluation metrics } \\
\cline { 2 - 4 } & Sensitivity & Specificity & Accuracy \\
\hline FCM+SVM & 0.643 & 0.5 & 0.555 \\
\hline PFCM+k-NN & 0.958 & 0.812 & 0.918 \\
\hline $\begin{array}{c}\text { Proposed } \\
\text { Cluster Deformable +NB }\end{array}$ & 0.986 & 0.546 & 0.790 \\
\hline
\end{tabular}

\section{ii) Analysis by k-fold variation}

The comparative analysis of the proposed scheme in SimBRATS database is presented in Table 2, and the analysis is done by varying the $\mathrm{K}$-fold values

Table 2: Comparative analysis with SimBRATS database for k-fold variation based on (a) Sensitivity, (b) specificity, and (c) accuracy using proposed Cluster deformable based fusion approach + NB For k=5

\begin{tabular}{|c|c|c|c|}
\hline \multirow{2}{*}{ Techniques used for Comparison } & \multicolumn{3}{|c|}{ Evaluation metrics } \\
\cline { 2 - 4 } & Sensitivity & specificity & Accuracy \\
\hline FCM+SVM & 0.841 & 0.598 & 0.711 \\
\hline PFCM + k-NN & 0.942 & 0.513 & 0.517 \\
\hline $\begin{array}{c}\text { Proposed Cluster Deformable } \\
\text { +NB }\end{array}$ & 0.987 & 0.607 & 0.848 \\
\hline
\end{tabular}

\section{Conclusion}

The RoC analysis of the comparative models against the proposed Cluster deformable $+\mathrm{NB}$ algorithm for the training data from SIMBRATS database is presented in Figure 3.5, for FPR $=0.2$, the comparative models, such as, FCM + SVM, and PFCM + k-NN have the TPR value of 0.824 , and 0.703, respectively. Meanwhile, the proposed Cluster deformable + NB algorithm provided better classification performance with the TPR value of 0.9 for the FPR $=0.2$.

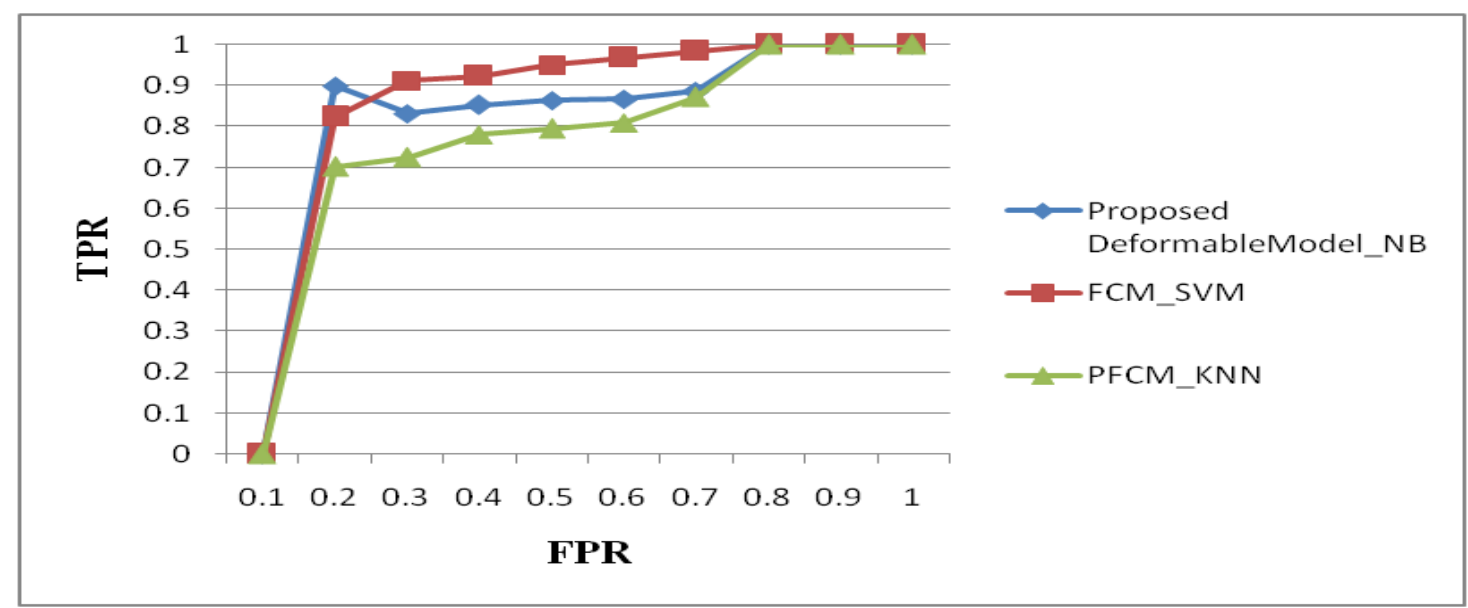

Fig 3.RoC analysis with SimBRATS database using proposed Cluster deformable based fusion approach + NB 


\section{References}

[1] Zhengwang Wu, Yanrong Guo, Sang Hyun Park, Yaozong Gao, Pei Dong, Seong-Whan Lee, and Dinggang Shen, "Robust Brain ROI Segmentation by Deformation Regression and Deformable Shape Model," Medical Image Analysis, vol. 43, pp. 198-213, 2017.

[2] Shoaib Amin Banday, and Ajaz Hussain Mir, "Statistical textural feature and deformable model based brain tumor segmentation and volume estimation," Multimedia Tools Application, vol. 76, no. 3, pp. 3809-3828, 2017.

[3]. Hala Ali, Mohammed Elmogy, Eman El-Daydamony, and Ahmed Atwan, "Multi-Resolution MRI Brain Image Segmentation Based On Morphological Pyramid and Fuzzy C-Mean Clustering," Arabian Journal for Science and Engineering, vol. 40, no. 11, pp. 3173-3185, November 2015.

[4] Ali Ahmadvand, Mohammad Reza Daliri, and Sayyed Mohammadreza Zahiri, "Segmentation of brain MR images using a proper combination of DCS based method with MRF," Multimedia Tools and Applications, pp. 1-18, 2017.

[5] R.Lavanyadevi, M.Machakowsalya, J.Nivethitha, and A.Niranjil Kumar, "Brain Tumor Classification and Segmentation in MRI Images using PNN", in proceedings on International Conference on Electrical, Instrumentation and Communication Engineering (ICEICE), 2017. 